\title{
Developing a Mobile Based Automated Testing Tool for Windows Phone 8
}

\author{
Albert Mayan. $\mathrm{J}^{1}$, Julian Menezes. $\mathrm{R}^{2} \&$ John Bruce. $\mathrm{E}^{3}$ \\ ${ }^{1}$ Associate Professor, Department of Computer Science and Engineering, Sathyabama University, India \\ ${ }^{2}$ P.G Scholar, Department of Information Technology, Sathyabama University, India \\ ${ }^{3}$ Associate Professor, Department of Computer Science and Engineering, Sathyabama University, India \\ Correspondence: Albert Mayan. J, Associate Professor, Department of Computer Science and Engineering, \\ Sathyabama University, $\quad$ Chennai-119, $\quad$ India. \\ albertmayan@gmail.com/mtechit2k13@gmail.com/johnbruce@sathyabamauniversity.ac.in
}

Received: February 6, 2015

Accepted: February 18, 2015

Online Published: July 25, 2015

doi:10.5539/mas.v9n8p91

URL: http://dx.doi.org/10.5539/mas.v9n8p91

\begin{abstract}
Smart phones, or Mobile phones are quickly fetching the essential computer and communication tool in people's life. Every phone has lots of applications and every application has its own different characteristics. Before producing these applications to the end user's use, the developer should confirm that the applications are working smoothly, sans any technical glitches, and user friendly in every feature, and for that we use testing tools to check the compatibility of the software by using test applications like Eggplant, silk test, etc. But nowadays, every tool is designed for the desktop environment. In this project an application is being proposed for the windows phones by which an end-user can install the application in the mobiles directly. After this work the end user will be able to know if the apps are working properly or not, this will help us to catch all the information by executing the data, detecting the type and the kernel. This application will help us to install any application on our windows phone by the other devices and will help to grab the elements in the present applications. It is much faster and user friendly, because it will work without the need of a desktop, and we can run some more test cases as well.
\end{abstract}

Keywords: testing, mobile testing, windows phone, phone testing, windows 8

\section{Introduction}

The Software development technologies, usually makes an apparent difference amidst functional testing and security testing. An automatic software testing is a function which verifies, that an executing component of source code is working well or not. Mobile application, also known as Mobile apps is a word to describe the application that was developed for low-powered devices. For, getting executed on the mobile, tab, computer, iPad or other electronic computer devices, a sort of application software is designed; it is termed as Mobile Apps or just a mobile app. An Application that creates a specific logic is preferred, if the aim is to interact with users, or to deliver an app that needs more work similar to a program than a web URL. A usual mobile structure differs in several conditions, the functionality of the device over the user's requirements, the accessing time, the operation in several locations and how it gets connected to the server in different ways.

The cell phone structure basically contains, a cell phone device, wired or wireless connectivity, backend server and an underlying network infrastructure. The application might be a stand-alone, or self-contained. The app depends on the capabilities of the hardware, or the application will run at the server end by remote and the mobile acts as a platform for interacting and viewing.

The mobile app will run on the server or the mobile device, the work of the mobile is dependent on the characteristics of the structure, the device potential and the behavior of the mobile applications. From the core business apps to the Enterprise environment there arises a requirement for an extra complicated situation to run these applications, which are providing mobile app interfaces in mobiles to communicate with the features, of some classic apps by the mobile clients.

Nowadays, Mobile devices have been built with the powerful Processors, Software execution abilities, storage, connectivity features, multi-media delivery with powerful Audio / Video, Accelerometers, Sensors and GPS 
facilities. The inbuilt camera in the phone, is not only helping the users to take a photo or record the video, but it also helps the user in face identification. The variety among the mobile apps which catches the imagination of the human brain is increased by the software development team. These applications are widely spread through several platforms such as Nokia, OVI, Lumia stores and the country service providers. The software which has been made for any specific platform may or may not run on all the devices that have been vended by the developers. Even if the platform is same, but the device compatibilities are different, then the apps will be unsuitable for other devices.

The mobile apps can be developed easily with a little bit of testing or without testing and dispersed without brief testing, because the mobile app developer focuses on the software functionalities instead of the device configuration, and approximately all the software testing is carried on emulators instead of the devices. The errors that exist in the app may harm the device, along with the application in which it is present. The requirement of the testing procedure assures the developer for the successfulness; venture to make the footprints in the mobile field. There are some determining studies that describe how to make a successful application.

The existing applications are all desktop based and not a mobile based application and it can test generalized windows 8 mobile applications through desktops alone. The main drawback lies in the complexity of the applications. Most of them are difficult to comprehend. As a result, this research work introduces a new mobile based automated testing tool for windows phone to resolve the above mentioned issues. In this proposed system, the automated testing tool will run on the windows phone itself. By utilizing the windows' simulation software (emulator) and Microsoft visual studio for Phone app development, the end-user can launch an application using another Windows application. The product thus created will be a mobile to mobile testing with both the testing applications and the application to be tested to be present on the same windows phone itself.

\section{Related Work}

Eggplant is a black-box GUI testing tool, which was given inception by Redstone Software in 2002. Redstone was acquired in 2008 by a UK-based company called Test Plant. Its approach uses image matching technology as opposed to looking at the object-level of the application being tested. This permits for a full system automation, of a system-under-test as compared to that of a solution related to an application. It also states that the technology, which is used to build the application to be tested is not a concern, nor does the system that the application runs on.

Currently, some of the apps which are available for downloads, can run on various mobile platforms such as NOKIA, and windows phones. However, the developers aren't making the public become aware, about the information, how the application will function on various platforms. Agile software is a group of development methods which is based on the growth of the apps, where the needs and the solution has been involved from the collaboration method amid cross-functional team. It is a theoretical framework which promotes interaction between the development cycles. The known author Dr. Jeff Sutherland and Ken Schwaber formed agile software development which is used by approximately, all the companies.

Kent Beck introduced a technique called Extreme programming, which is a well known process in agile process, that is used for lightweight, low-risk, predictable, flexible and scientific way for software development. In this process the client or user works with the development team and gets a high priority for software testing on iteration basis.

The most lightweight methods for developing the software are crystal methodology. It has some types such as crystal clear, crystal orange, crystal yellow, by which characteristics are obtained by project priorities and team size. The crystal method needed policy standard, certain roles and products. This method is applied to the team size of 6-8 members. This method mainly focuses on the requirement of the client or user instead of the artifacts. The author in has proposed a new technique that is known as FDD (Feature Driven Development). This technique combines some practice in one methodology. These are all the techniques which are functioning through the client viewpoint.

The methodology for documenting and modeling the software is based on practice. It is based on the collection of principles and the values of Agile modeling and it is more flexible. This technique is the supplement of the XP (Extreme processing), Scrum and RUP (Rational Unified process). 


\section{Proposed Work}

\subsection{Architecture}

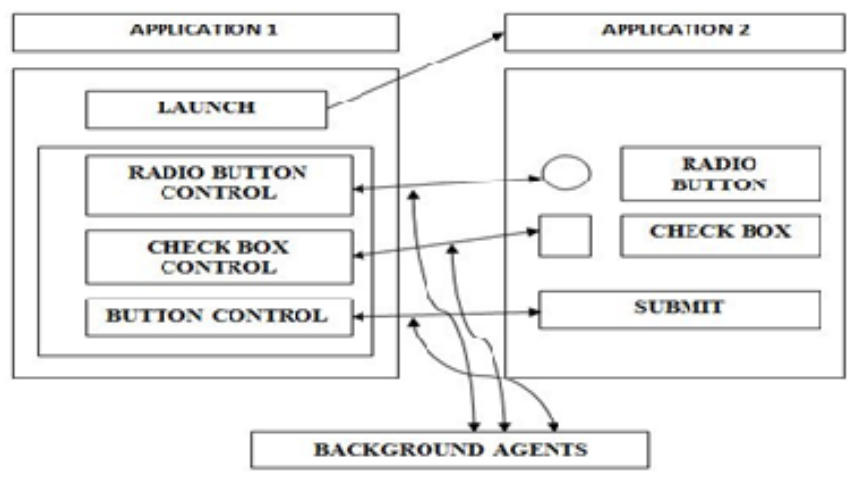

Figure 1. Proposed System Architecture

\subsection{Proposed Architecture Overview}

The proposed system developed a mobile based automated testing tool for handling various installed applications in the windows mobile. The proposed testing tool uses the following components such as Microsoft visual studio 2013, Windows Phone 8, Software Development Kit 8. Initially, a source code is developed to launch one application using another application in windows operating system version 8.1. After that, a foreground as well as a background application is created. The foreground windows application is installed in one Windows PC or Windows phone, as mentioned in Fig.1. Then, the background application is installed on the same windows phone. After installing foreground and background applications, the user launches foreground application on the Windows phone. Using a URI (Uniform Resource Identifier) an end-user can launch the background application. Similarly, with the help of user Interface (UI) the user can also launch various background applications. The proposed application launches any other application present in our windows 8 phone and it grabs the objects present in the particular application.

\subsection{Implementation}

This stage describes the details about the implementation of our proposed mobile based automated testing tool. Our proposed automated tool utilizes Emulator for connecting Windows Phone with PC. Detail description of Emulator is given below:

\subsubsection{Emulator}

The proposed tool is developed with the great help of an EMULATOR. The use of these emulators are extremely useful in the initial stages of the development of the application, because they allow quick and efficient checking of the progress, of the application. An emulator is a system that runs software from one environment to another without changing the integrity of the software itself. It duplicates the features and works of a real system.

Some advantages of using an emulator are:

- All applications can be launched and subjected to test on an emulator, without the need for investing in a new mobile handset for various versions of windows phone.

- Emulators are mostly available for free, and we can also perform stress, UI and performance testing using it.

- Emulators are easy to use without the need to have knowledge of any complex features in it.

- The version of the Emulator involved in the project is "Emulator WVGA 512MB".

\subsection{Working Procedure}

\subsubsection{Launching One Windows form Application}

Initially two simple windows form applications are created, one application is Installed in windows 8 phone and another application is installed on windows PC using Microsoft visual studio 2013 and windows emulator. After that, one windows form application is launched from another windows window form application. It is made quite easier for us to understand, how one application launches another application, whether it may be desktop to 
desktop, or mobile based application to another mobile based application.

\subsubsection{Connecting One Application to another Application}

Generally, the function call helps the user to access two functions simultaneously. The Function call works based on the function command which starts the execution, when the users calls the respected function. After launching an application, we can successfully call the functions of the elements of another application from the windows forms application.

\subsubsection{Automating the Tests Performed on Windows Forms.}

This module describes the details of the automated testing process using windows forms. The next approach adopted was to automate all the tests which was performed by calling the functions of the elements, of the other foreground application from the background application. The automated process was done by looping method. The Looping method helps the testing process by making a loop in the background application/form for all the functions in the foreground application/form. It was done by launching a windows 8 phone application on emulator 8/8.1.

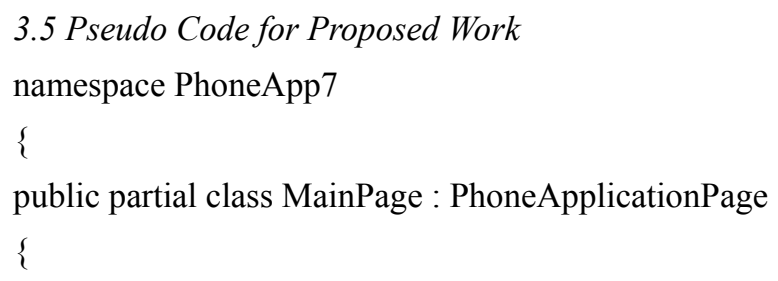

Public async void Button_Click_1(object sender, RoutedEventArgsrea)

\{ // Launch URI.

awaitWindows.System.Launcher.LaunchUriAsync(newSystem.Uri("PhoneApp7"));

\section{\} \} \}}

\section{Results and Discussions}

To evaluate the performance of the proposed automated Testing tool, a series of experiments on the installed applications in windows 8 phone were conducted. In these experiments, the proposed automated tool was implemented and evaluated, and it requires the following configurations. The Table 1 below lists out all the Hardware and the Software required for the development of the Mobile Testing Tool.

Table 1. Hardware \& Software Requirements

\begin{tabular}{ll}
\hline S.No & REQUIREMENTS \\
\hline 1. & Windows 8 Mobile Phone. \\
2. & Processor: Intel(R) COre(TM) i7-3770K CPU @ 3.90 GHz. \\
& RAM: 8.00 GB Hard Disk Drive: 500GB. \\
& Operating System: Windows 8.1 x64 (Professional). \\
3. & Microsoft Visual Studio 2013. \\
& Software Development Kit 8. \\
4. & Emulator WVGA 512MB \\
\hline
\end{tabular}

\subsection{Experiments}

By using the above mentioned components the proposed testing tool can efficiently test the applications. 


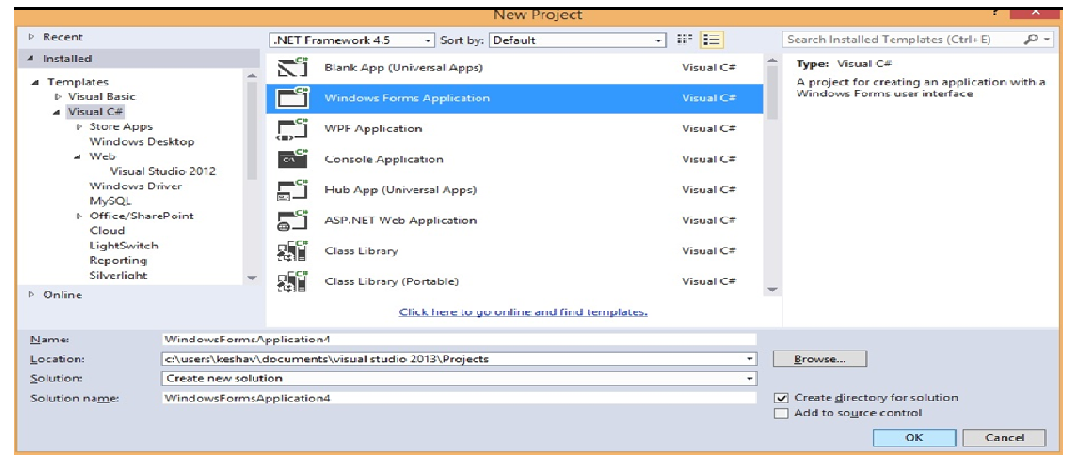

Figure 2. Windows Forms Application (Foreground)

In the first step, the Windows forms application in .Net framework 4.5 with the Visual C\# is chosen. This framework is used for launching one application from another application.

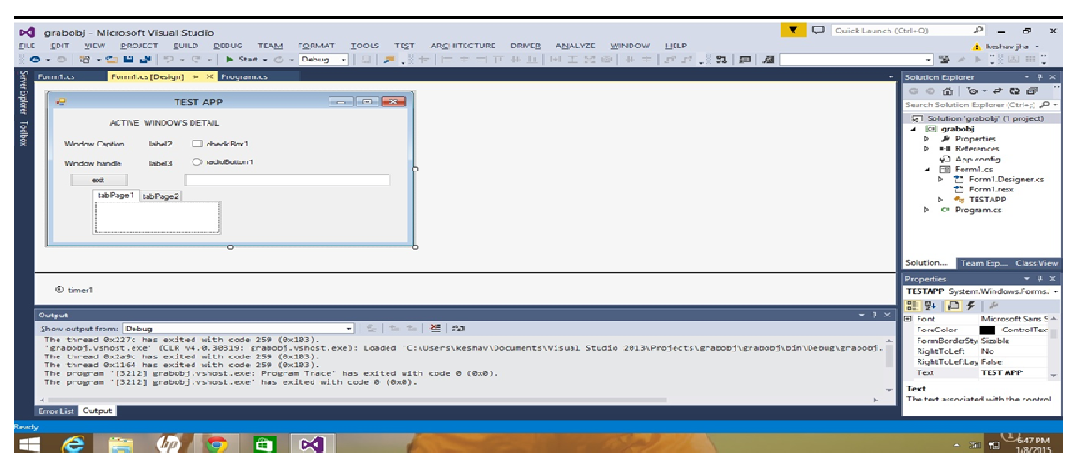

Figure 3. Test Application

After choosing the Windows form application the developer is in need to draw a set up for the Test application for both the devices, (Mobile and Desktop) as well. In this step the developers generate, the internal Modeling for the software which is communicating with other designed software.

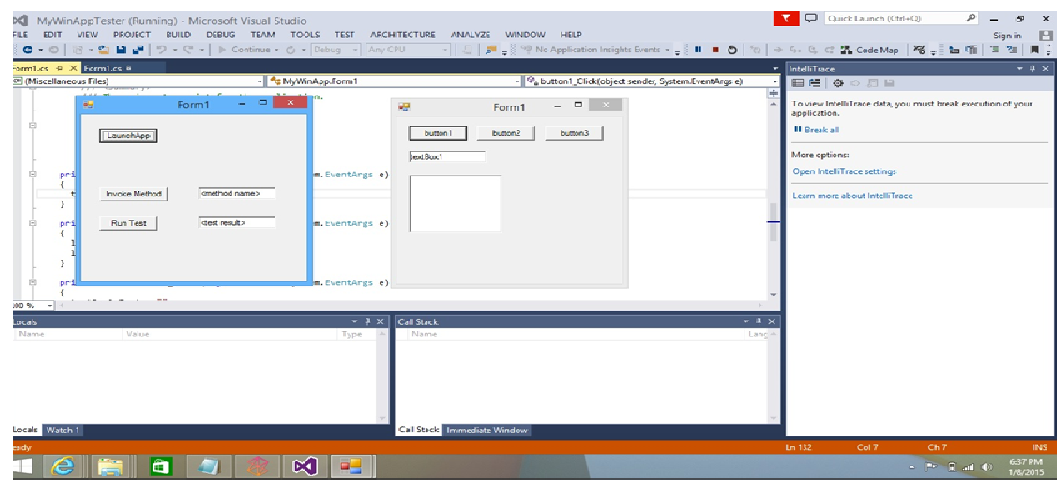

Figure 4. Launching Foreground application

Both the software Designs are finalized and the software is processed for installing in the Windows phone and Desktop. These softwares will communicate with each other for reading the contents of the other device. 


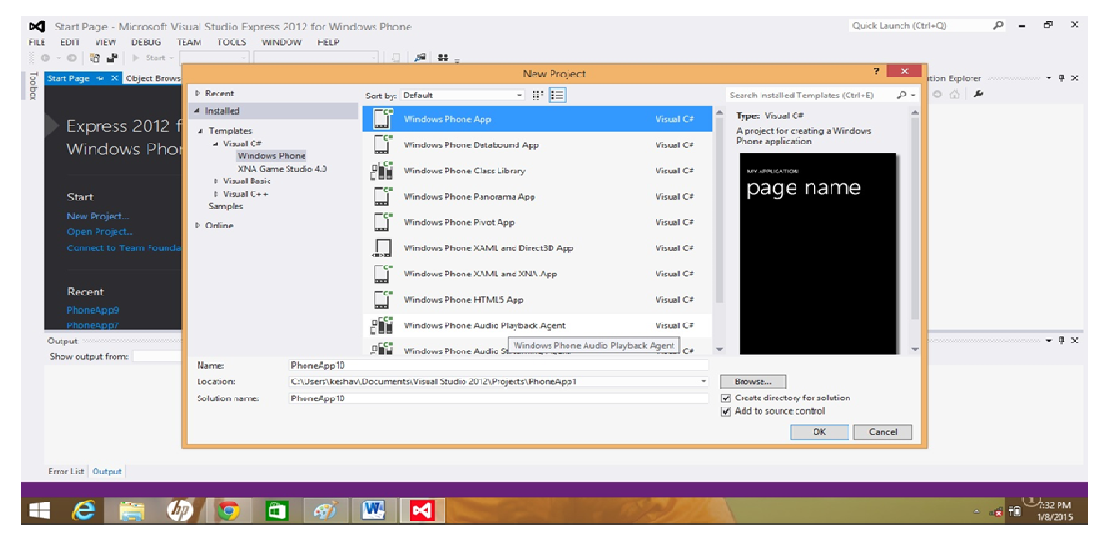

Figure 5. Connecting Background Application using URI

The Apps created by .Net framework 4.5 is accessed on the Mobile side (background) which will communicate with the (Desktop side) foreground application. The installed application shows the status for the other installed application as well. The (PC side) foreground installed application has to start the new page title or the page name.

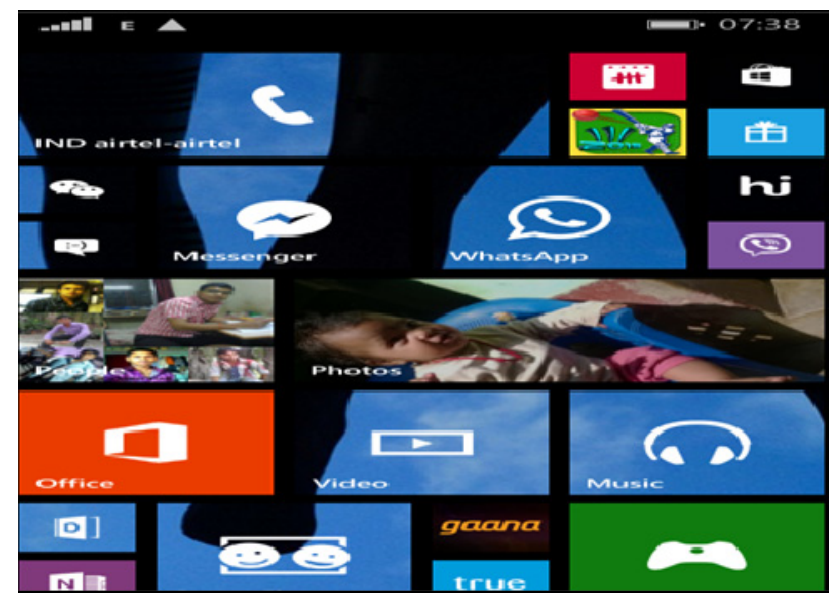

Figure 6. Launching Background Application

The installed application is getting connected with the background application in Windows phone. After launching an application, we can successfully call the functions of the elements of another application from the tester windows forms application.

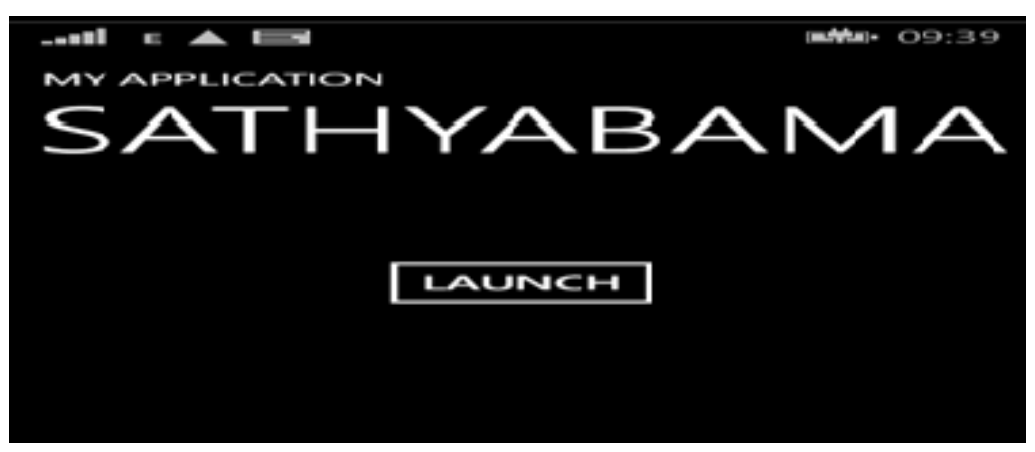

Figure 7. Expected Output 
Finally the communication has been established over both ends. By using this application the end-user can launch any other application installed in windows mobile and after launching that app, it will grab all the objects and elements present in the launched application.

\subsection{Performance Evaluation}

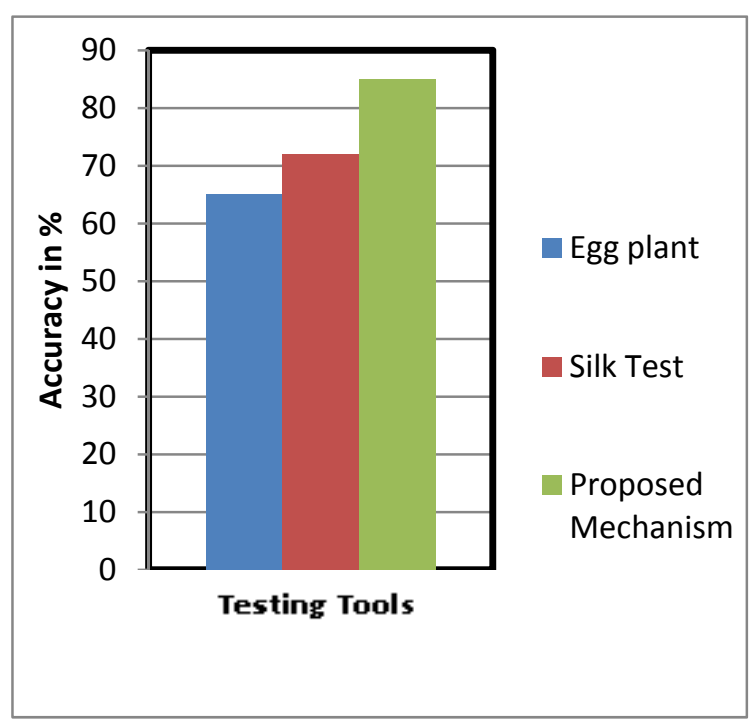

Figure 8. Accuracy of Testing Tools

The Figure 8 shows the testing accuracy of proposed mobile based Automated Testing Tool. It does not depend on the use of a Hardware apart from the Mobile itself. It tailors the need of an organization for one unique purpose, unlike other testing tools. It can be launched from within a Mobile and takes less or no time to trigger the testing process. As a result, our proposed testing mechanism achieves maximum testing accuracy than other traditional testing tools like eggplant, silk test, etc.

\section{Conclusion}

In this research, the proposed System implements a mobile based automated testing to efficiently test the applications in Windows phone 8. This system utilized the mentioned tools for testing the foreground application by using the background application on windows phone. In this proposed automated testing tool, the user can clutch the techniques and substances present in an active window on a desktop screen, the same technique is used to perform the action similar for an active window in a Windows application, but the way is unclear on how to install this application from desktop to windows phone. Utilizing the proposed work it is possible to do a direct installation directly in the windows phone through the desktop environment.

\section{References}

A Guide to Emulators . Retrieved from http://mobiforge.com

Agile Modelling. Retrieved from http://en.wikipedia.org/wiki/Agile_Modeling

Albert, M. J., \& Ravi, T. (2015). Structural Software Testing: Hybrid Algorithm for Optimal Test Sequence Selection during Regression Testing. International Journal of Engineering and Technology (IJET), 7(1), 2015.

Crystal Clear (2013). Retrieved from http://en.wikipedia.org/wiki/Crystal_Clear_(software_development)

Extreme Programming. (1996). Retrieved from http://www.extremeprogramming.org

Feature Driven Development. (2009). Retrieved from http://en.wikipedia.org/wiki/Feature_Driven_Development

Hyo-Eun, E., \& Lee, S. W. (2013). Human-centered software development methodology in mobile computing environment: agent supported agile approach. Eom and Lee EURASIP. Journal on Wireless Communications and Networking, 111. Retrieved from http://jwcn.eurasipjournals.com/content/2013/1/111

IEEE. (2000). IEEE Standard for Software Test Documentation. IEEE Std 829. 
Ken, S., \& Sutherland, J. (2011). The Definitive Guide to Scrum: The Rules of the Game.

Kent, B., (2004). Extreme Programming explained (2nd ed.). Addison- Wesley, Boston.

Malloy, A. D., Varshney, U., \& Snow, A. P. (2002). Supporting mobile commerce applications using dependable wireless networks. Mobile Networks and Applications, 7(3), 225-234.

Mobile Stats. Retrieved from http://www.slideshare.net/vaibhavkubadia75/mobile-web-vs-mobile apps-27540693? from_search=1

Ondrej, K., Jakub, J., \& Dalibor, J. (2011). Use of Mobile Phones as Intelligent Sensors for Sound Input Analysis and Sleep State Detection. ISSN 1424-8220. http://dx.doi.org/10.3390/s110606037

Selvam, R., \& Karthikeyani, V. (2011). Mobile Software Testing-Automated Test Case Design Strategies. International Journal on Computer Science and Engineering (IJCSE), 3(4). ISSN: 0975-3397

Testlabs Blog. (2010). Top 10 Tips for Testing iPhone Applications. Retrieved September, 2010, from http://blog.testlabs.com/search/label/iPhone

Wooldridge, D., \& Michael, S. (2010). The business of iPhone app development: Making and marketing apps that succeed. Apress.

\section{Copyrights}

Copyright for this article is retained by the author(s), with first publication rights granted to the journal.

This is an open-access article distributed under the terms and conditions of the Creative Commons Attribution license (http://creativecommons.org/licenses/by/3.0/). 